Herzschr Elektrophys 2013 · 24:1

DOI 10.1007/s00399-013-0261-y

Online publiziert: 19. April 2013

(c) Springer-Verlag Berlin Heidelberg 2013

Carsten W. Israel

Evangelisches Krankenhaus Bielefeld, Klinik für Innere Medizin - Kardiologie \& Angiologie, Bielefeld, Deutschland

\title{
Interventionen bei Vorhofflimmern
}

\section{Nichtpharmakologische Rhythmuskontrolle und Schlaganfallprävention}

prophylaxe bei Vorhofflimmern betrifft. Nach dem Nachweis einer Nicht-Unterlegenheit in zwei randomisierten Studien (PROTECT AF und PREVAIL) gewinnt diese Therapie zunehmend Eingang in die tägliche Praxis.

Im zweiten Teil des Themenschwerpunktes dieser Ausgabe von Herzschrittmachertherapie + Elektrophysiologie werden daher Hinweise gegeben, für welche Patienten die Implantation eines Vorhofohr-Okkluders eine hilfreiche Therapie sein könnte und wie die Implantation verschiedener Devices genau durchgeführt wird. Auch chirurgische Verfahren werden dargestellt, die ja im Zusammenhang mit Herzoperationen aus anderen Gründen (Bypass, Klappen) schon lange durchgeführt werden.

Zusätzlich zum Themenschwerpunkt werden in dieser Ausgabe von Herzschrittmachertherapie + Elektrophysiologie neue Rubriken vorgestellt: In der Rubrik $E H$ $R A$-Corner sollen regelmäßig Neuigkeiten und Aktivitäten der europäischen Rhythmologie berichtet werden, in einer separaten Rubrik Neuigkeiten aus der AG1 Rhythmologie der DGK. Einmal im Jahr versuchen wir, die „Highlights“ verschiedener Bereiche der Elektrophysiologie aus dem vergangenen Jahr zu bewerten, um mit ein paar Wochen Abstand abzuschätzen, welche Publikationen des letzten Jahres wichtig waren und in der Studienlandschaft in Erinnerung bleiben sollten.

Den Autoren der Artikel in dieser Ausgabe von Herzschrittmachertherapie + Elektrophysiologie sei herzlich für die interessanten, klar strukturierten Arbeiten ge- dankt. Ich wünsche den Lesern viel Spaß bei der Lektüre!

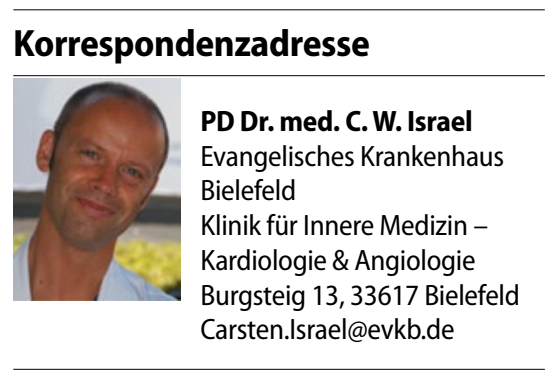

Der Vorhofohrverschluss mit Devices stellt ein neueres interventionelles Therapieverfahren dar, das die Schlaganfall- 